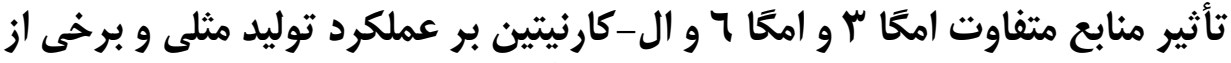

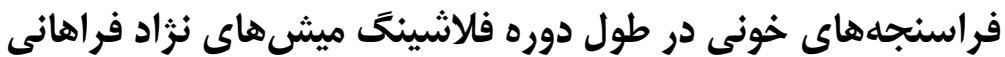

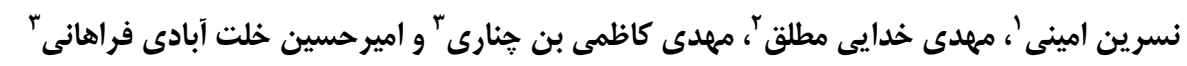

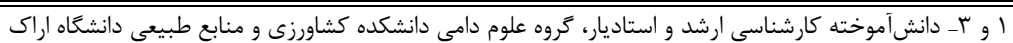

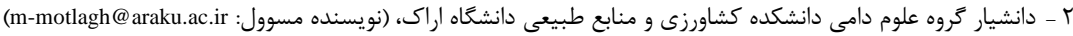 \\ تاريخ دريافت: 9F/N/TD
}

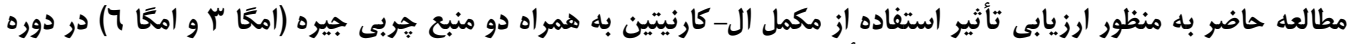

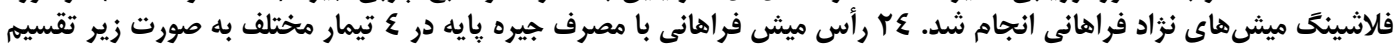

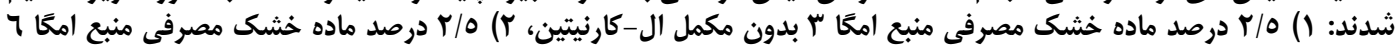

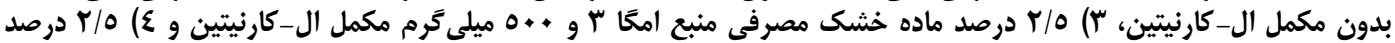

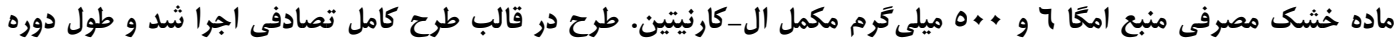

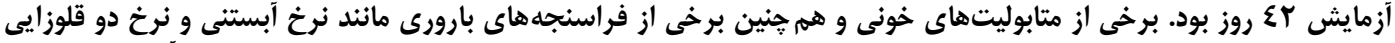

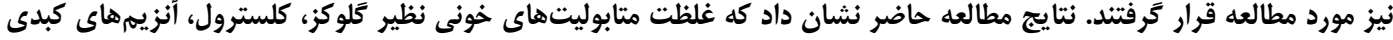

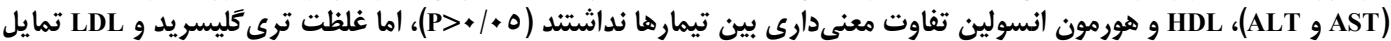

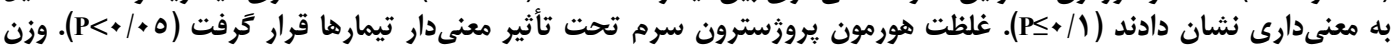

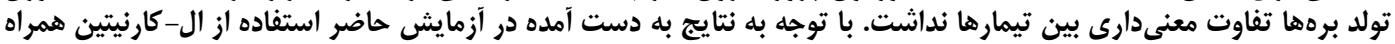

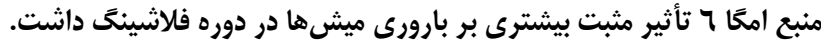

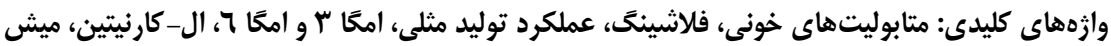

غيرفعال) و ال-كارنيتين (شكل فعال) (1). كبـد، كليـهـ و مغـز

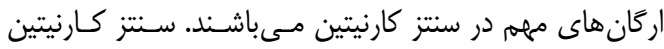

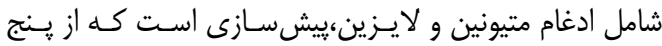

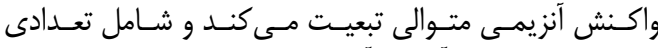
كوفاكتور مانند يون آهن و آسكوربات است آنست (1). عملكرد اوليـهـ

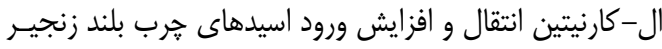

به ماتريكس ميتو كندرى براى بتا-اكسيداسيون است است (1).

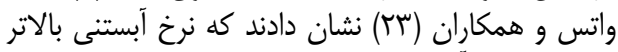

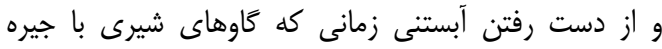

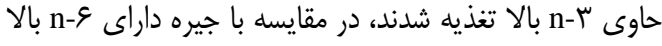

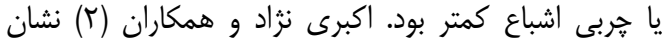

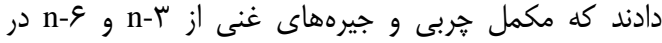

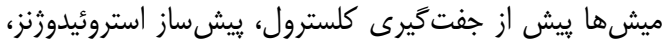
LDL

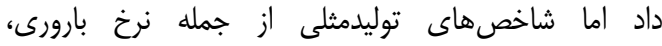

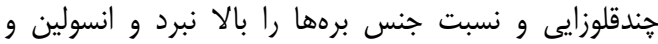

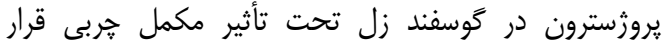
نداشتند.

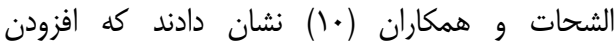

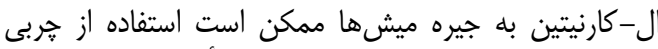

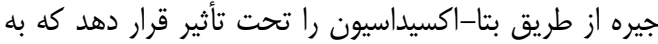

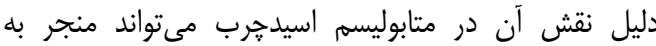

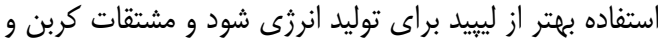

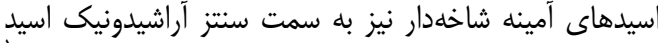

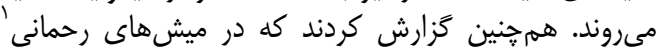

بازده توليد مثل نقش اساسى در تعيين بازده اقتصادى

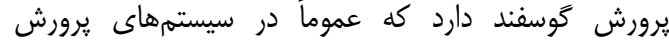

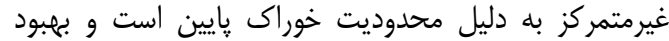

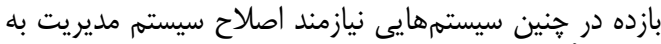

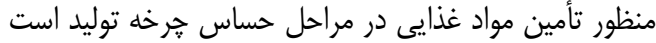

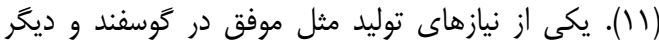

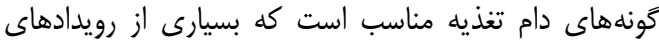

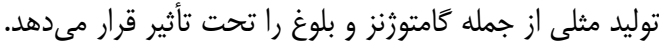

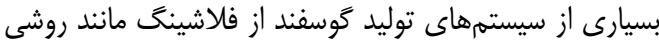

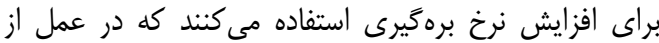

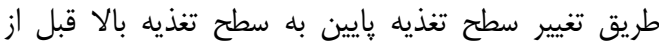
جفت گيرى صورت مى

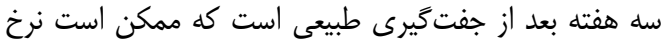

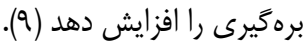

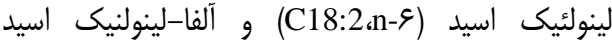

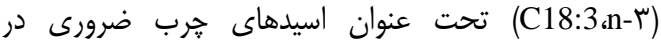

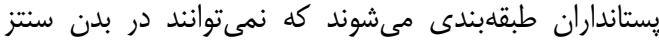

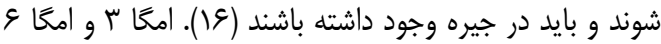

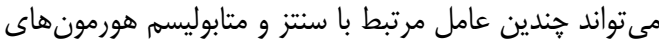

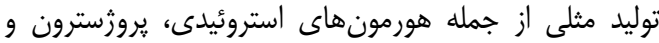

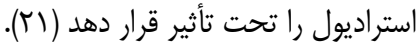

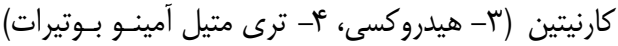

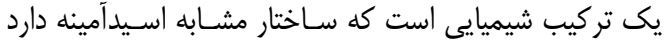
كه بـه شـكل دو ايزومــر وجـود دارد: دى - كـارنيتين (شـكل 


\section{مواد و روشها}

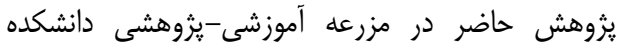

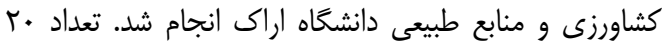

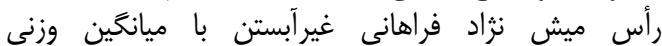

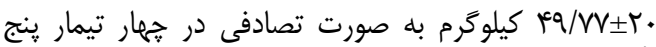

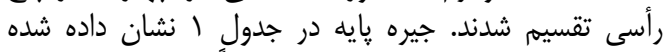

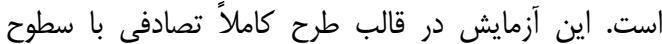

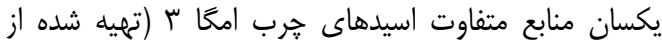

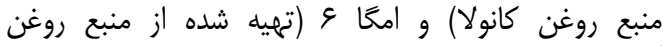

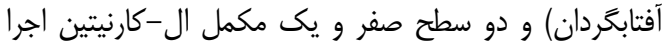

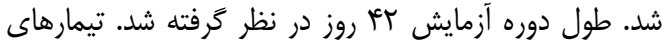

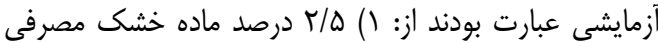

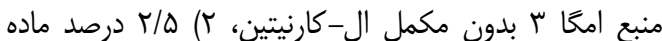

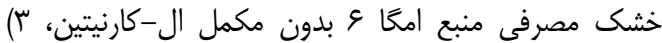

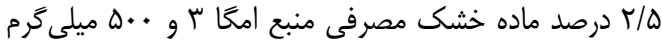

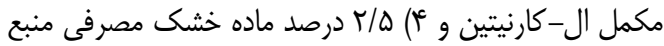

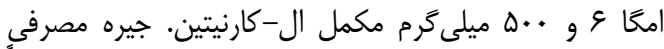

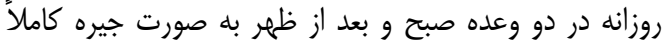

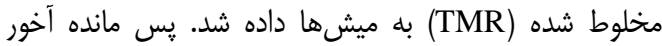

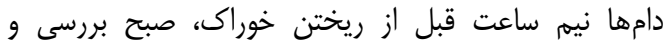

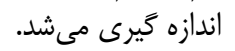

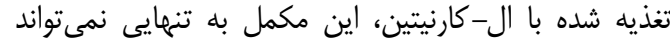

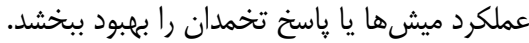

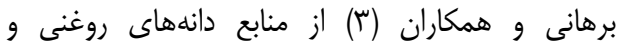

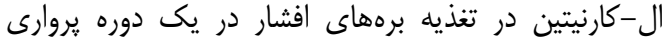

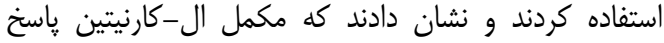
برهها را به تغذيه جيرههاى حاوى داند دانه سويا و دانه كانه كانولان

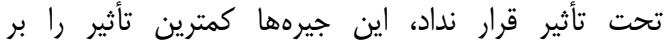

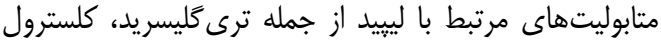

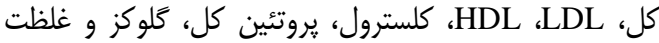

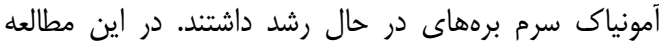

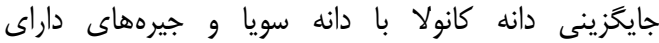
ال-كارنيتين تأثيرى بر خوراك بانه مصرفى، قابليت هضم دوانه مواد

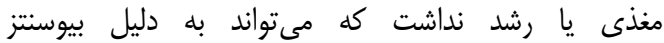

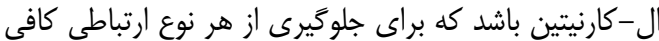

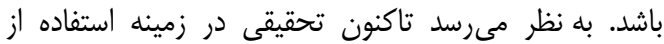

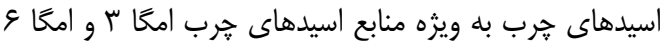

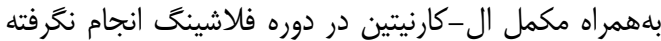

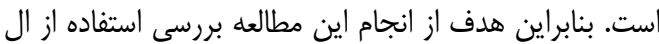

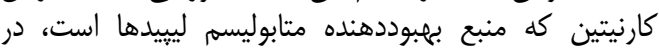

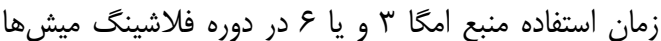
خواهد بود.

جدول ا- اجزاى تشكيل دهنده جيره پايه براساس درصد از ماده خشك به همراه تركيبات شيميايى جيره يايه Table1. Ingredient composition of basal diet based on percent of dry matter with chemical composition basal diet

\begin{tabular}{|c|c|}
\hline درصد ماده خشك & مواد خوراكى \\
\hline ry & يونجه \\
\hline$r \Delta / \Delta$ & جو \\
\hline IV & سبوس \\
\hline IV & كاه \\
\hline$r / \Delta$ & كنجاله سويا \\
\hline$\cdot / \mu$ & مكمل ويتامينـ \\
\hline.$/ 1$ & نمك \\
\hline.$/ 1$ & دى كلسيم فسفات (DCP) \\
\hline$r / \Delta$ & مكمل تربى \\
\hline & تركيبات شيميايى جيره پايه \\
\hline$r / r$ & انرزى (مكاكالرى در كيلوكرم ماده خشك) \\
\hline $1 \% / 4$ & يروتئين(درصد در ماده خشك) \\
\hline
\end{tabular}

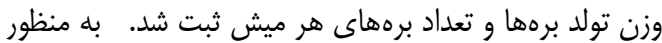

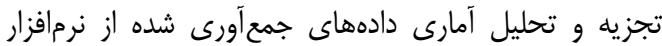
SAS

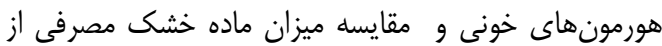

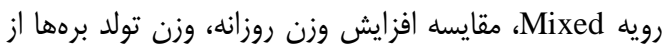

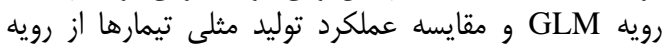

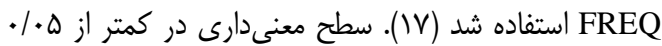

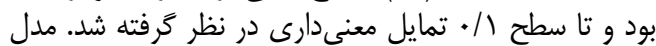

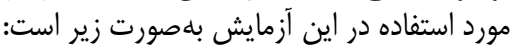
$Y_{i j}=\mu+T_{i j}+e_{i j} \quad i=1, \ldots, a ; j=1, \ldots, a$ كه در اين مدل Yij: متغير وابسته، ب: ميانكين كلى،

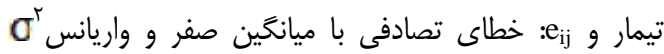

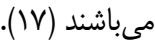

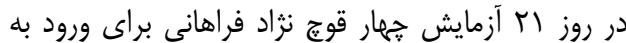

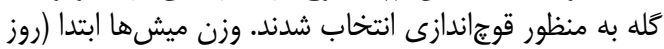

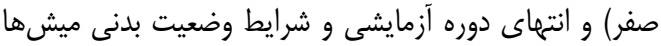

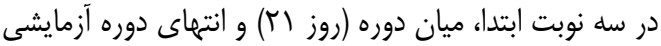

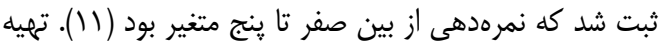

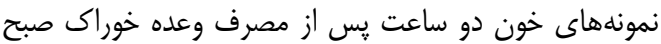

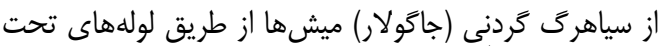

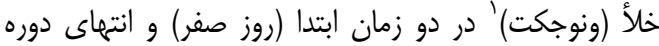

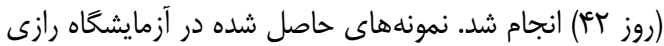

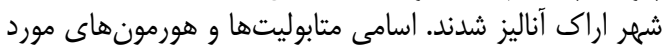

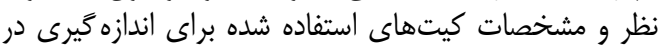

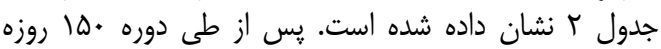
آبستنى اطلاعات زايش از جمله وزن واده ميش در إن زمان دوره زايش،

1- Clot Activator 
جدول r- متابوليتها و مشخصات كيتهاى آزمايشخاهى استفاده شده براى اندازمخيرى Table2. Metabolites and traits of laboratory kits used for measurement

\begin{tabular}{|c|c|c|}
\hline شماره كيت & نام كيت & متابوليت \\
\hline $9 \mu \cdots 1$ & پارس أزمون & كلوكز \\
\hline $9 T \cdot 1$ & هارس آزمون & ترى كليسريد \\
\hline $94 . .9$ & پيارس آزمون & كلسترول \\
\hline $94 \cdot .0$ & يارس آزمون & آسيارتات آمينوترانسفراز (AST) \\
\hline 9 q... & يارس آزمون & آلانين آمينوترانسفراز (ALT) \\
\hline $9 \% \cdot \Delta$ & بيشتاز & HDL \\
\hline $9 \mu \cdot{ }^{c}$ & يِيشتاز & LDL \\
\hline$E L A=r V K r_{F} F^{c}$ & Monobind & انسولين \\
\hline $\mathrm{ELA}=\mathrm{L} \backslash \mathrm{K} \mathrm{K}^{\mathrm{C}} \mathrm{DF}$ & Monobind & يروزسترون \\
\hline
\end{tabular}

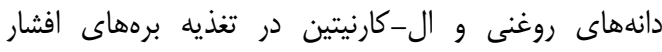

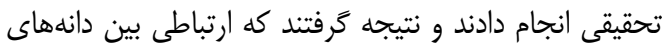

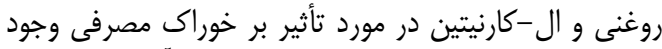

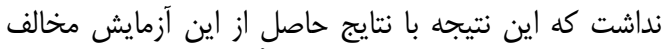

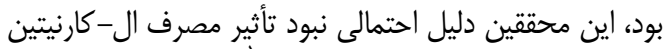

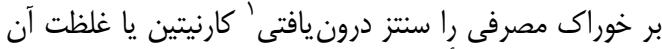

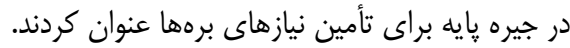

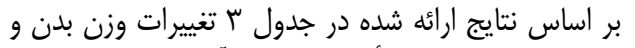

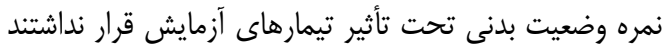

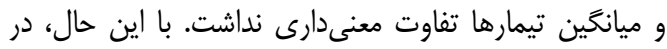

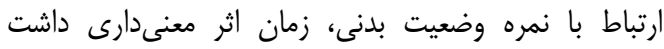

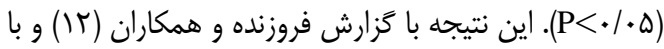

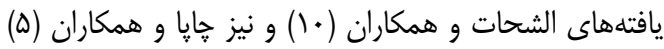

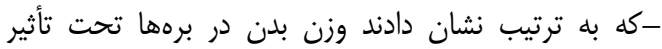

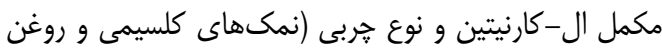

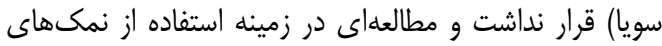

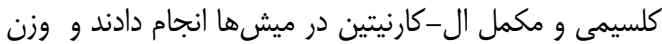

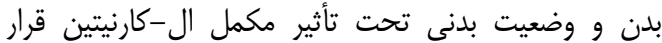

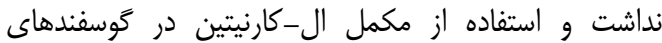

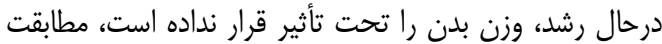
داشت. - ماده خدال

\section{نتايج و بحث \\ خوراك مصرفى، عملكرد و امتياز وضعيت بدنى}

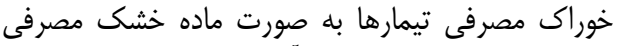

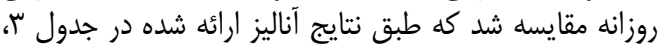

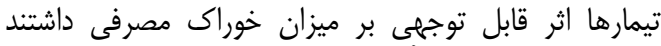

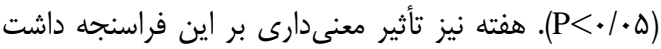

$(P<+1 \cdot \Delta)$

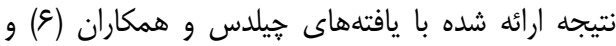

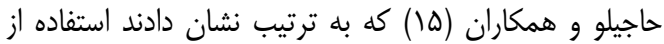

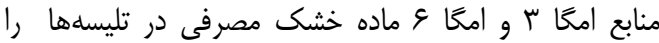

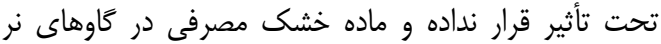

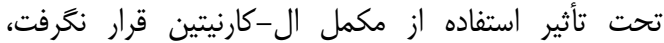

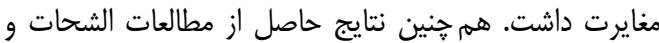

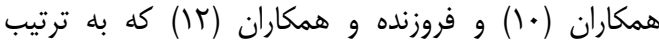

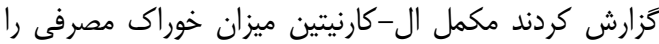

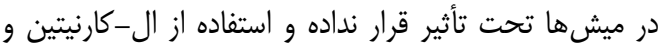

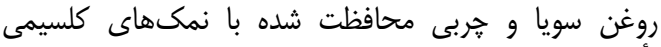

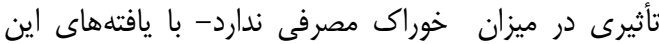
مطالعه ناساز كار بود. برهانى و همكاران (ب) در خصوص استفاد استاده از منابع

جدول بـ تأثير منابع متفاوت امكا س و امكا و و و ال-كارنيتين بر ماده خشى مصرفى، تغييرات وزن، افزايش وزن روزانه و وضعيت

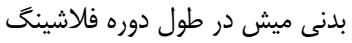

Table 3. The effect of different sources of omega-3 and omega-6 fatty acids and L-carnitine on dry matter Intake, weight changes, average daily gain and body condition of ewe in during flushing period

\begin{tabular}{|c|c|c|c|c|c|c|c|c|c|}
\hline \multicolumn{4}{|l|}{ P Value } & \multirow[b]{2}{*}{ SEM } & \multicolumn{4}{|c|}{ تيمار } & \multirow[t]{2}{*}{ فراسنجه } \\
\hline \multirow[t]{2}{*}{ وزن روز صفر } & تيمار × زمان & زمان & تيمار & & r & $r$ & $r$ & 1 & \\
\hline & $\cdot / \cdots 1$ & $\cdot / \cdots+1$ &.$/ \cdots 1$ & $\cdot 1 \cdots 9$ & $1 / 99^{d}$ & $1 / \Lambda \gamma^{b}$ & $1 / 9 \cdot^{a}$ & $1 / \wedge \Delta^{c}$ & ماده خشك مصرفى (كيلوكَرم/ روز \\
\hline - / $\Delta$ VGa & - & - & ./VEGT & $1 / \mp \wedge$ & $\Delta / 9 D$ & V/AT & $V / T V$ & $V / F F$ & \\
\hline •/DVT" & - & - & $\cdot / V \in \Delta$. & $.1 \cdot 4$ & $f$ &.$/ 1 \wedge$ &.$/ \mathrm{Vr}$ & . IV & (5رم) (5 ) \\
\hline - & $\cdot / \cdot v$ & $|\cdots+|$ &.$/ \mathrm{rA}$ & $\cdot / \mu$ & $\Gamma / \Delta \Delta$ & 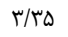 & $\mu / \Delta$ & $\Gamma / \Gamma \Delta$ & امتياز وضعيت بدنى \\
\hline \multicolumn{10}{|c|}{ 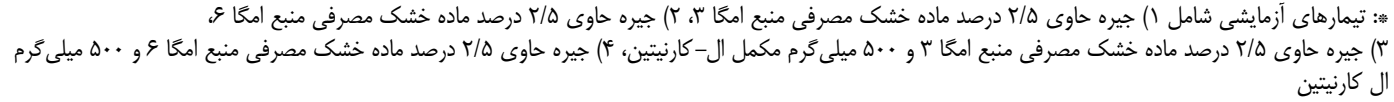 } \\
\hline \multicolumn{5}{|c|}{ 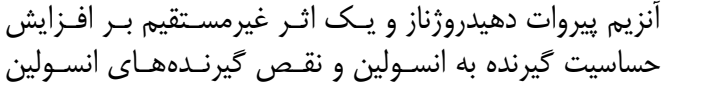 } & \multicolumn{5}{|c|}{ 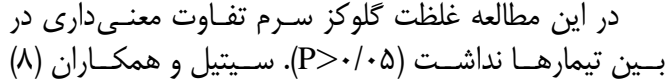 } \\
\hline \multirow{2}{*}{\multicolumn{5}{|c|}{ 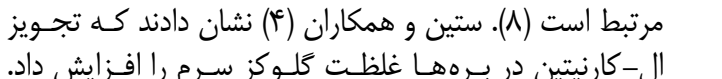 }} & \multirow{2}{*}{\multicolumn{5}{|c|}{ 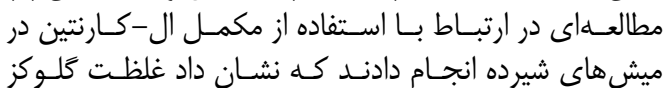 }} \\
\hline \multirow{2}{*}{\multicolumn{5}{|c|}{ 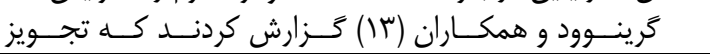 }} & & & & & \\
\hline & & & & & \multicolumn{5}{|c|}{ يلاسما تحت تأثير اين مكمل كاهش يافت. مكانيسم احتمـالى } \\
\hline
\end{tabular}

1- De-novo 
در اين مطالعه با توجه به اين كه جيره بايه در تيمارهاى تواى

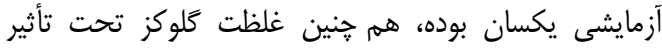
معنى دار تيمارها قرار نداشت بنابراين تغييرى در سطح آنسان انسولين

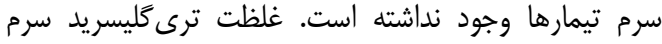

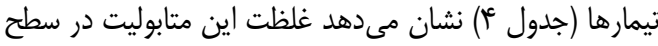

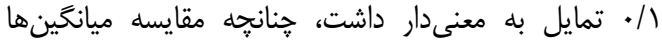

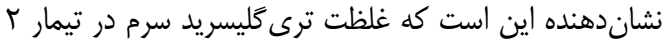

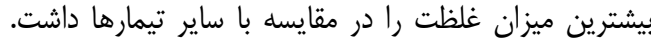

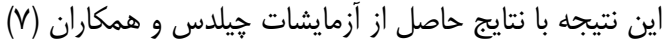

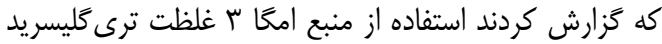

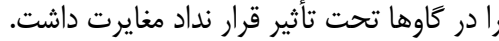

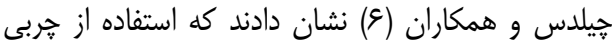

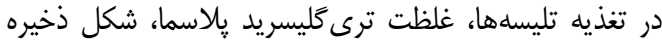

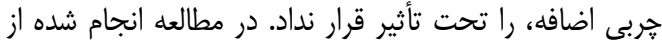

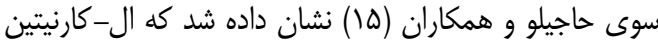

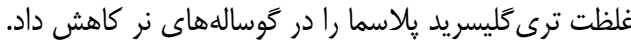

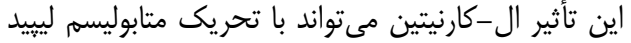

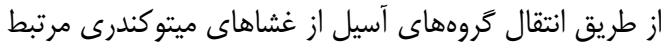

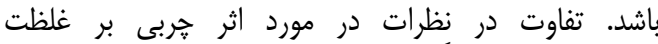

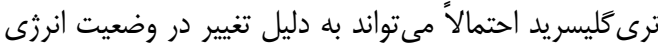

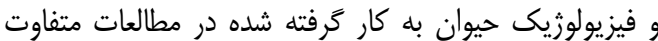

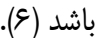

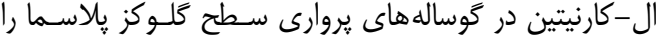

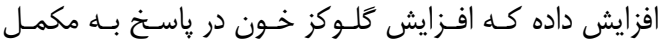

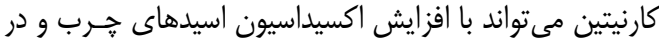

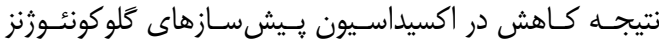

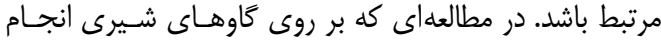

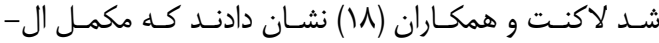

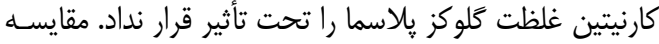

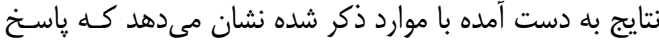

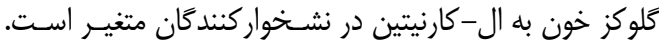

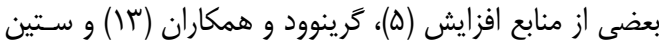

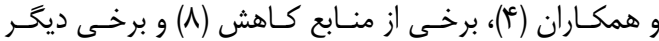

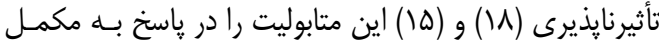

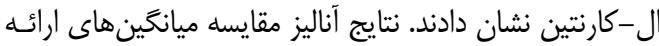

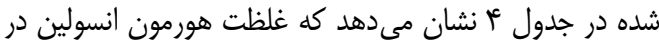

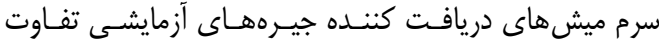

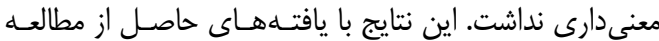

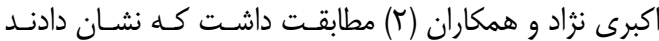

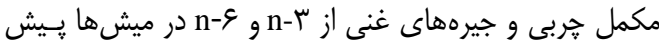

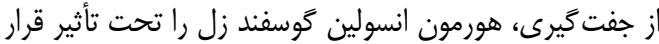

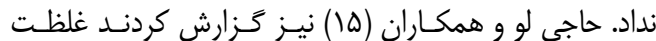

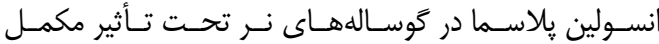

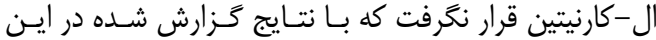

مطالعه مشابة بود.

جدول عأ- تأثير منابع متفاوت امخا س و امكا \& و ال-كارنيتين بر متابوليتها و هورمونهاى خونى ميش در طول دوره فلاشينگ Table 4. The effect of different sources of omega-3 and omega-6 fatty acids and L-carnitine on blood metabolites and hormones of ewe during flushing period

\begin{tabular}{|c|c|c|c|c|c|c|c|c|}
\hline \multirow{2}{*}{$\begin{array}{l}\text { P Value } \\
\text { تيمار×زمان }\end{array}$} & \multirow[b]{2}{*}{ زمان } & \multirow[b]{2}{*}{ تيمار } & \multirow{2}{*}{ SEM } & \multicolumn{4}{|c|}{ 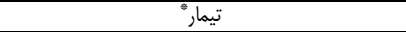 } & \multirow{2}{*}{ 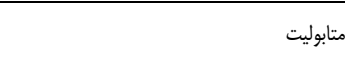 } \\
\hline & & & & t & r & r & 1 & \\
\hline$\cdot / t \cdot 4$ & $\cdot / 41 \wedge 9$ &.$/ 1499$ & $\Delta / \cdot r$ & $V T / \mu$ & $9 \pi / 9$ & $V \varphi / \Delta$ & $91 / T^{5}$ & كلوكز (ميلى گرم/ دسىليتر) \\
\hline.$/ 19.9$ &.$/ 8114$ & $\cdot /$ HEV & 1/ar & $8 / 10$ & $r / \Delta 1$ & ع/r/ & $T / 1 Q$ & انسولين (ميكرو واحد بين المللى/ ميلىليتر) \\
\hline.$/ 99 \vee \Delta$ & . & $\cdot / \cdots 1$ & $r / \& g$ & $t r / T^{2}$ & $1 \mathrm{~V} / \mathrm{a}$ & 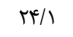 & $10 / 1$ & ترى كليسريد (ميلى گرم/ دسىليتر) \\
\hline.$/ 9 Y V D$ &.$/ \cdots r$ & $\cdot / \& \wedge \Delta \varepsilon$ & $r / v \wedge$ & $v e / \varepsilon$ & $V N / T$ & $v e / \Delta$ & $\mathrm{V} / \mathrm{A}$ & كلسترول (ميلى \\
\hline.$/ 10$ & $* \ldots+$ & $\cdot / 1 \cdot r \cdot$ & V/DF & $\mathrm{IV} / \mathrm{T}$ & $r / / 8$ & $r M / \cdot$ & $r T / \cdot$ & لييويروتئين كم קخالى (ميلى گرم/ دسىليتر) \\
\hline$\cdot / \& \wedge \vee \wedge$ & $\cdot 1 \cdots 1$ & $\cdot / \Lambda \mid \Delta S$ & $r / \cdot$ & $\Delta \cdot / 8$ & $F N /$ & $\mathrm{F} / \mathrm{T}$ & $f \Delta / \mathrm{V}$ & لييويروتئين يرجخالى (ميلى گرم/ د \\
\hline | & $\cdot / \cdot M$ &.$/ 11 \cdot 9$ & $\mid V / F 9$ & TrT/ATI & (rg/OT & س & $199 / .9$ & آسياراتات آمينوترانسفراز (واحد بين المللى/ ليتر) \\
\hline $.1 .8 \mathrm{TV}$ &.$/ .914$ & . /Arsq & $r / \Lambda)$ & th/TH & $r \mid / 9 q$ & tI/at & W/Ar & آلانين آمينوترانسفراز (واحد بين المللى/ ليتر) \\
\hline$\cdot / V \mu \cdot \cdot$ & • &.$/ . \mp \Delta \Delta$ &.$/ 4 \wedge$ & $r / r 1^{a b}$ & 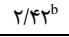 & $r / \cdot V^{b}$ & $r / \cdot r^{a}$ & يروزسترون (نانو گرم/ ميلىليتر) \\
\hline
\end{tabular}

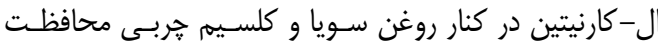

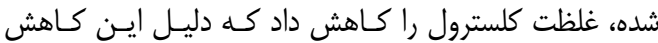

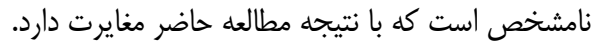

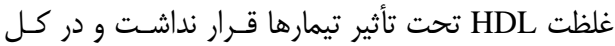

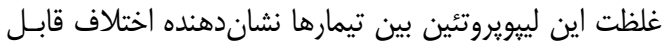

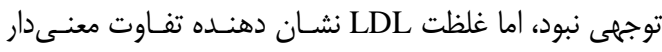

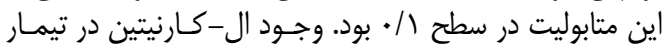

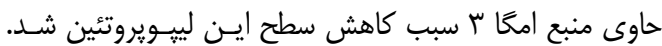

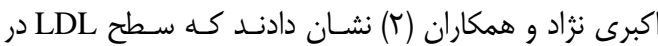

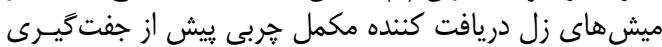

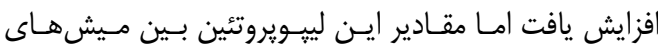

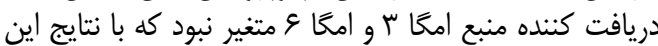

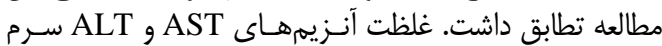

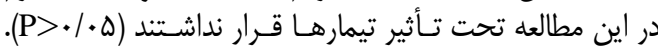

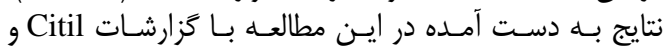

غلظت كلسترول سرم تحت تأثير تيمارهاى آزمايشى قـرار

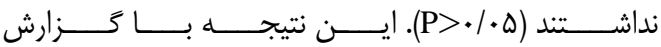

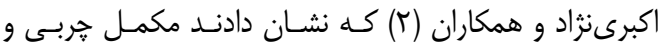

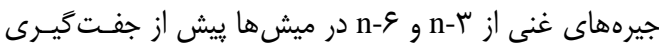

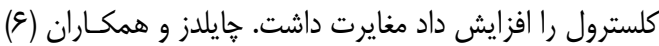

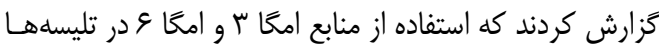

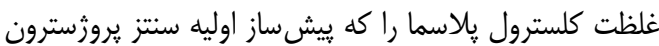

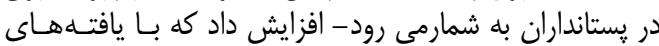

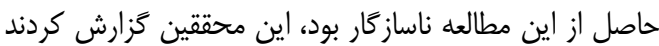

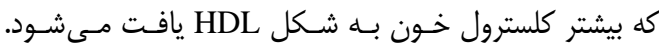

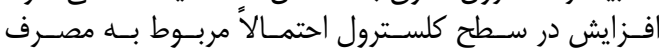

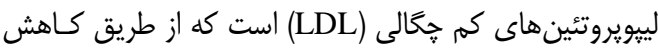

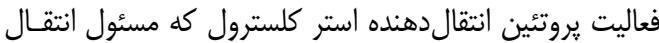

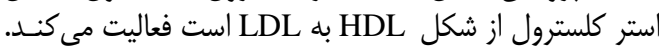

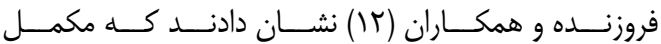




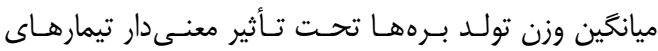

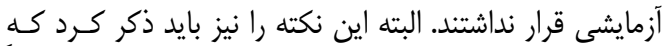

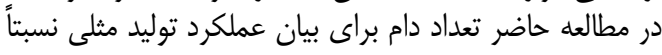

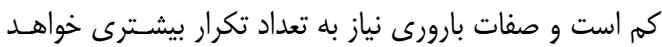

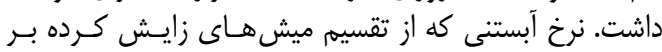

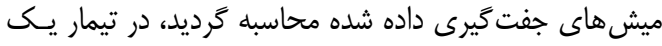

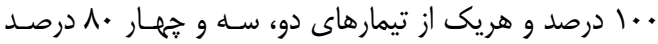

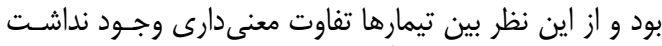

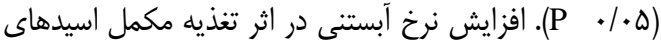

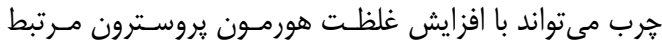

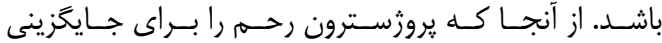

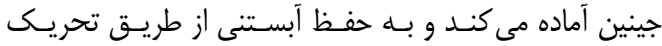

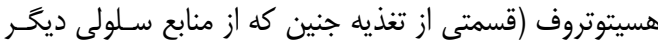

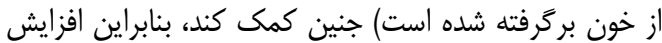

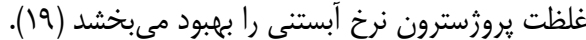

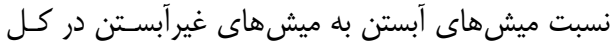

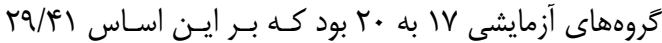

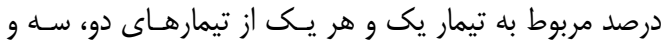

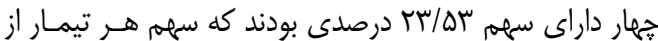

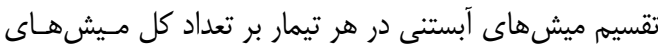

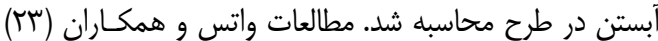

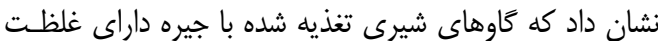

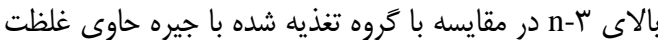

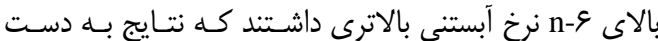

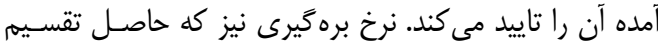

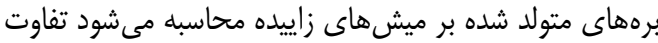

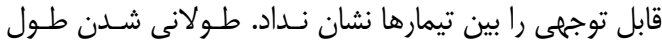

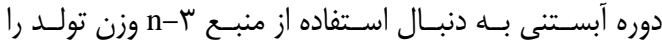

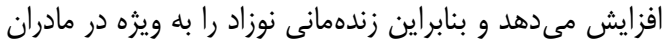

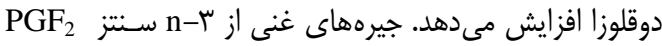

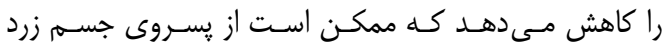

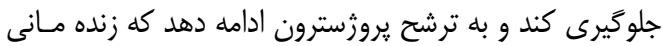

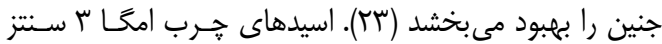
PGF $2 \alpha$

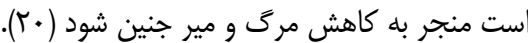

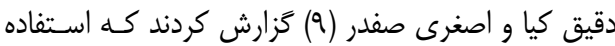

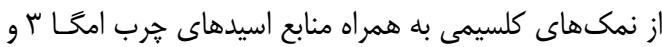

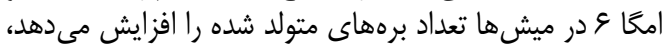

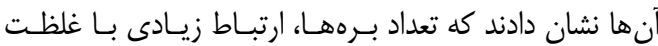

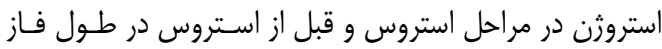

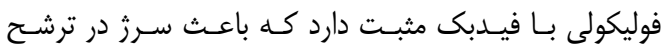

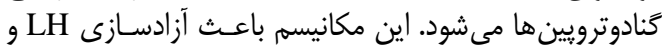

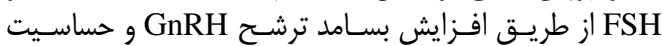

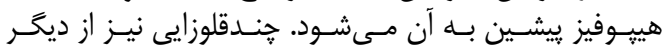

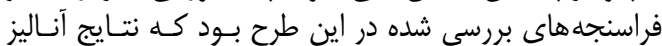

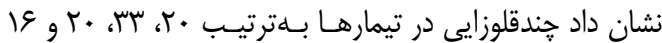

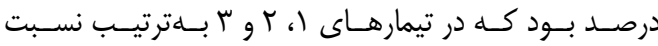

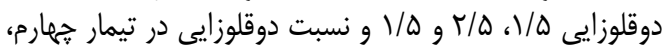

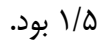

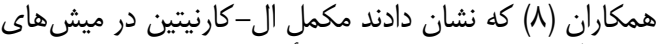

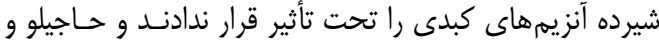

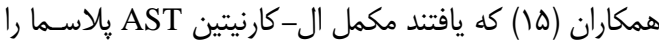

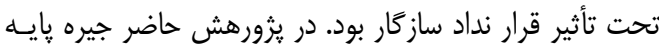

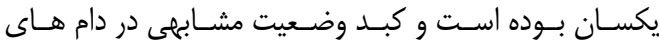

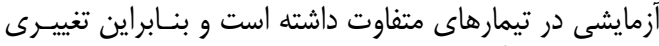

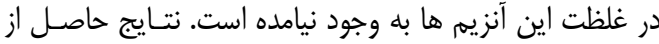

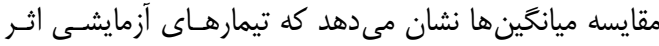

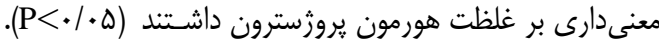

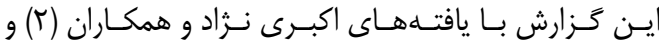

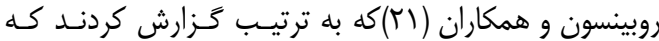

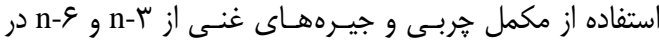

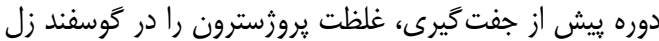

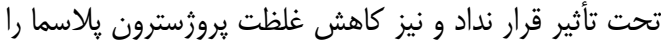

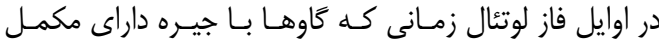

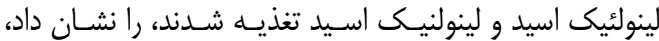

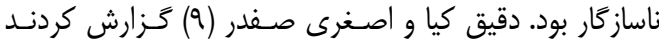

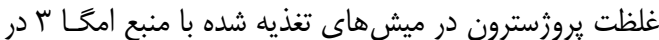

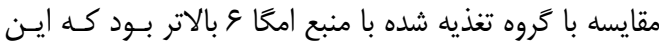

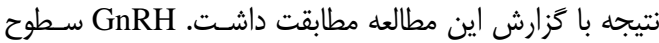

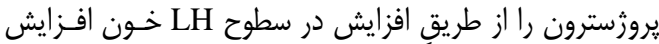

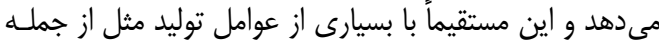

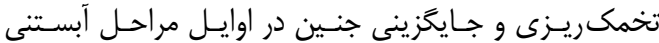

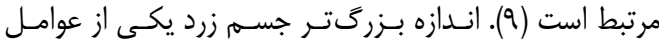

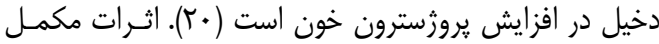

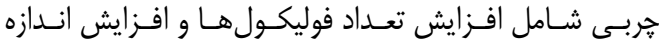

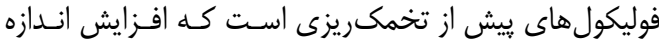

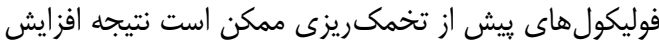

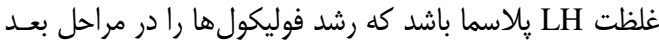

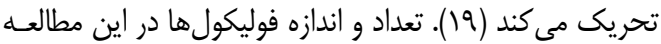

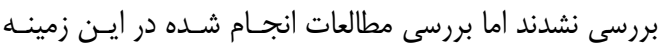

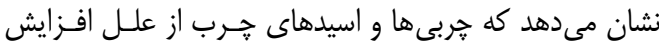

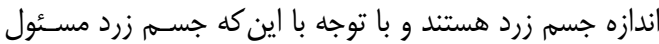

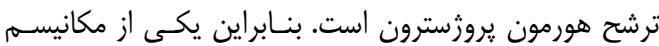

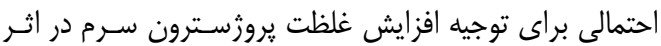

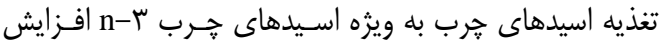

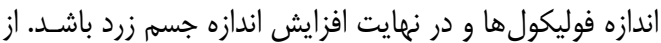

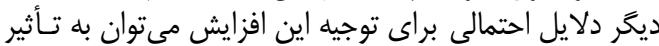

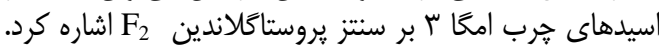

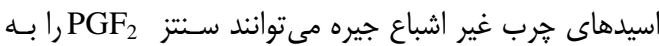

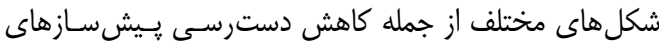

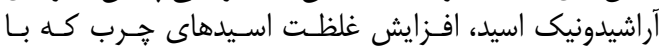

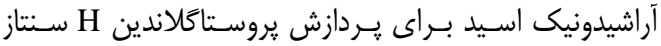

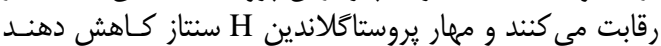

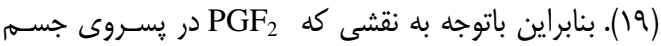

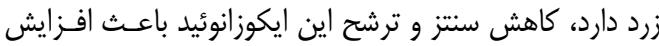

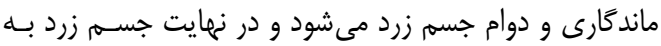

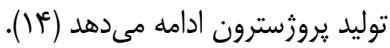

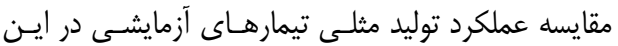

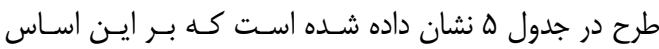


جدول ه- تأثير منابع متفاوت امًا ب و امًا و و ال-كارنيتين بر برخى از عملكرد توليدمثلى ميش در طول دوره فلاشينگ Table5. The effect of different sources of omega-3 and omega-6 fatty acids and L-carnitine on some reproductive performances of ewe in during flushing period

\begin{tabular}{|c|c|c|c|c|c|c|}
\hline تعداد برهها & نرخ برهيرى & جندقلوزايى & ترخ آبستنى بين & (تعداد دام آبستن) & 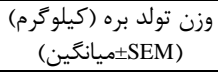 & تيمار" \\
\hline 9 & $1 / T \cdot$ & $r \cdot$ & $r q / 4)$ & $\Delta / \Delta$ & $r / \Lambda r_{ \pm} \cdot / T^{\prime}$ & 1 \\
\hline 4 & $1 / 0$. & سז & TH/DT & $f / Q$ & $F / T V_{ \pm} \cdot / r \Delta$ & r \\
\hline$\Delta$ & $1 / \pi \Delta$ & $r$. & $r / \Delta r$ & $f / \Delta$ & $\Gamma / \vee q_{ \pm} \cdot / \mathcal{F} \cdot$ & r \\
\hline 4 & $1 / 0$. & 19 & TH/QT & $f / \Delta$ & $r / q_{ \pm} \cdot / q q$ & f \\
\hline
\end{tabular}

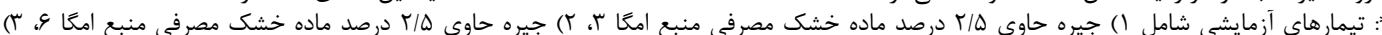

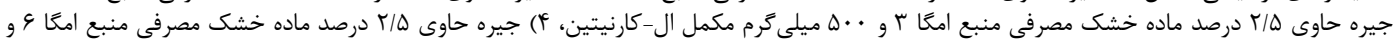

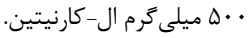

1. Abuzaid, A.A. 2010. Variation of carnitine concentrations in Angus beef. M.Sc. Dissertation, Iowa State University.

2. Akbarinejad, V., A. Niasari-Naslaji, H. Mahmoudzadeh and M. Mohajer. 2012. Effects of diets enriched in different sources of fatty acids on reproductive performance of Zel sheep. Veterinary Research, 13: 310-316.

3. Borhani, M., A.D. Foroozandeh, S.M. Nasrollahi and H.R. Amini. 2015. Effect of oilseed sources and L-carnitine administration on growth, feed intake, feed digestibility and blood metabolites of Afshari lambs. Agricultural Science, 49: 1-5.

4. Cetin, M., M. Petek, U. Polat and A. Yalcin. 2003. Effects of dietary carnitine supplementation on plasma carnitine and some serum biochemical parameters in lambs. Revuede Medecine Veterinaire, 154: 195-198.

5. Chapa, A.M., J.M. Fernandez, T.W. White, L.D. Bunting, L.R. Gentry, T.L. Ward and S.A. Blum. 1998. Influence of intravenous L-carnitine administration in sheep preceeding on oral urea drench. Animal Science, 76: 2930-2937.

6. Childs, S., C.O. Lynch, A.A. Hennessy, C. Stanton, D.C. Wathes, M.G. Diskin and D.A. Kenny. 2008. Effect of dietary enrichment with either n-3 or n-6 fatty acids on systemic metabolite and hormone concentration and ovarian function in heifers. Animal Science, 2: 883-893.

7. Childs, S., Z. Cheng, A.A. Hennessy, J.M. Sreenan, D.C. Wathes, Z. Cheng, C. Stanton, M.G. Diskin and D.A. Kenny. 2008. Effect of level of dietary n-3 polyunsaturated fatty acid supplementation on systemic and tissue fatty acid concentrations and on selected reproductive variables in cattle. Theriogenology, 70: 595-611.

8. Citil, M., M. Karapehlivan, H.M. Erdogan and R. Yucayurt. 2009. Effect of orally administered Lcarnitine on selected biochemical. Small Ruminant Research, 81: 174-177.

9. Daghigh Kia, H. and A.H. Asgari Safdar. 2015. Effects of calcium salts of fatty acids (CSFA) with different profiles $\left(\mathrm{w}_{3}\right.$ and $\left.\mathrm{w}_{6}\right)$ during the flushing period on reproductive performance of 'Afshari' ewes. Small Ruminant Research, 126: 1-8.

10. El-Shahat, K.H. and A.M. Abo-Elmaaty. 2010. The effect of dietary supplementation with calcium salts of long chain fatty acids and/or L-carnitine on ovarian activity of Rahmani ewes. Animal Reproduction Science, 117: 78-82.

11. Esmaeili-Zadeh, A., S.R. Miraei-Ashtiani and M. Akbari Gharaei. 2002. Effects of ewe live weight and body condition at mating on fertility and lambing season of Kurdy sheep in extensive production system. Pajouhesh \& Sazandegi, 61: 8-16 (In Persian).

12. Foroozandeh, A.D., M. Shahzeydi, H.R. Amini, S.M. Nasrollahi and G.R. Ghalamkari. 2014. The effect of fat type and L-carnitine administration on growth, feed digestibility and blood metabolites of growing Afshari lambs. Livestock Science, 164: 67-71.

13. Greenwood, R.H., E.C. Titgemeyer, G.L. Stokka, J.S. Drouillard and C.A. Loset. 2001. Effects of Lcarnitine on nitrogen retention and blood metabolites of growing steers and performances of finishing steer. Animal Science, 79: 254-260.

14. Gulliver, C.E., M.A. Friend, B.J. King and E.H. Clayton. 2012. The role of omega-3 polyunsaturated fatty acids in reproduction of sheep and cattle. Animal Reproduction Science, 131: 9- 22.

15. Hajilou, M., M. Dehghan-Banadaky, A. Zali and K. Rezayazdi. 2013. The effects of dietary Lcarnitine and rumen-protected choline on growth performance, carcass characteristics and blood and rumen metabolites of Holstein young bulls. Applied Animal Research, 42: 98-96.

16. Hughes, J. 2011. The effect of dietary omega-3 and -6 polyunsaturated fatty acids on ovine ovarian function and the pre-implanation embryo. Ph.D. Dissertation, Nottingham University.

17. Kaps, M. and W.R. Lamberson. 2004. Biostatistics for animal science. 7 th., page 459.

18. Lacount, D.W., J.K. Drackely and G.J. Weigel. 1995. Responses of dairy cows during early lactation to ruminal or abomasal administration of L-carnitine. Dairy Science, 78: 1824-1836.

19. Mattos, R., C.R. Staples and W.W. Thatcher. 2000. Effects of dietary fatty acids on reproduction in ruminants. Reproduction and Fertility, 5: 38-45.

20. Petit, H.V. and H. Twagiramungu. 2006. Conception rate and reproductive Function of dairy cows fed different fat sources. Theriogenology, 66: 1316-1324.

21. Robinson, R.S., P.G. Pushpakumara, Z. Cheng, A.R. Peters, D.R. Abayasekara and D.C. Wathes 2002. Effects of dietary polyunsaturated fatty acids on ovarian and uterine function in lactating dairy cows. Reproduction, 124: 119-131.

22. Soofi-Siawash, R. and H. Janmohammadi. 2011. Animal nutrition, 6 edn, Amidi Publications, Iran, 908 pp (In Persian).

23. Wathes, D.C., D.R. Abayasekara and R.J. Aitken. 2007. Polyunsaturated fatty acids in male and female reproduction. Biology of Reproduction, 77: 190-201. 


\title{
The Effect of Different Sources of Omega-3 and Omega-6 Fatty Acids and L-carnitine on Reproductive Parameters and Some Blood Metabolites in Farahani Ewe during Flushing Period
}

\author{
Nasrin Amini ${ }^{1}$, Mahdi Khodaei Motlagh ${ }^{2}$, Mehdi Kazemi-Bonchenari ${ }^{2}$ and \\ Amir Hossein KhaltAbadi-Farahani ${ }^{2}$ \\ 1 and 3- M.Sc. Student Assistant Professor, Arak University \\ 2- Assistant Professor, Arak University (Corresponding author: m-motlagh@araku.ac.ir) \\ Received: November 16, $2015 \quad$ Accepted: January 30, 2016
}

\begin{abstract}
The present study was conducted to evaluate the effect of L-carnitine supplementation accompanied with two sources of fat supplemented diets ( $\omega 3$ and $\omega 6)$ on flushing period of Farahani ewes. 20 Farahani ewes were allocated in four different treatments which were as follow: 1) $2.5 \%$ dry matter intake $\omega 3$ source without L-carnitine 2) $2.5 \%$ dry matter intake $\omega 6$ source without L-carnitine 3) $2.5 \%$ dry matter intake $\omega 3$ source with $500 \mathrm{mg}$ L-carnitine 4) $2.5 \%$ dry matter intake $\omega 6$ source with $500 \mathrm{mg} \mathrm{L}$-carnitine. The experiment was conducted as completely randomized design and the study was lasted 42 days. Some blood metabolites were studied and fertility parameters were considered as well. The results of the present study showed that blood metabolites concentrations such as glucose, cholesterol, hepatics enzymes (AST, ALT), HDL lipoprotein and insulin were not significantly different among the treatments $(\mathrm{P}<0.05)$. However serum triglyceride and LDL lipoprotein showed to have a tendency to be significant (P $₫) .1)$. Serum progesterone concentration was affected by treatments $(\mathrm{P}<0.05)$. Lamb birth weights were not significantly different among treatments. According to the obtained results sources of omega 3 and 6 had positive impact but the accompanying of L-carnitine with omega- 6 showed to have more positive effect on ewes in flushing period.
\end{abstract}

Keywords: Blood metabolites, Ewe, Flushing, Fertility performance, L-Carnitine, Omega 3 and Omega 6 